\title{
New mothers' awareness of newborn screening, and their attitudes to the retention and use of screening samples for research purposes
}

\author{
ANGELA DAVEY, DAVINA FRENCH, HUGH DAWKINS \& PETER O'LEARY
}

\begin{abstract}
Aim: To explore new mothers' knowledge of newborn screening, and their attitudes towards issues surrounding sample retention and the potential for blood screening samples to be used for research.
\end{abstract}

Methods: A self-administered mail survey was sent to women who gave birth in Perth, Western Australia during January 2005. A total of 600 women completed the survey.

Results: It was found that women were aware of newborn screening, however desired further information in order to acquire a more comprehensive knowledge of the test. Further, women reported discomfort with the long-term storage of cards, but they were supportive of using blood samples for medical research, contingent upon the samples being de-identified and parental consent provided.

Conclusions: New mothers need to be provided with comprehensive information about the newborn screening test at a time which is conducive for the assimilation of this information. In addition, whilst supporting health related research using newborn screening samples, new mothers are keen for ethical issues to be sufficiently addressed prior to samples being systematically stored for extended periods of time. 


\section{Introduction}

For several decades the genetic blood screening of newborns to detect inborn errors of metabolism has been recognised as a valuable component of neonatal care in many developed countries around the world. ${ }^{12}$ The early detection of these disorders has proven an effective means by which interventions can be implemented to significantly reduce morbidity, mortality and associated disabilities. ${ }^{34}$ Publicly funded newborn screening programs have been operating in Australia since 1964, and although participation is entirely voluntary, there is high public participation in most programs 1 , enabling them to be cost effective. ${ }^{6}$

At present Australian newborn screening programs are working towards developing a nationally consistent approach to the retention of newborn screening cards, and secondary uses of the blood samples derived from these cards. At the time of this study in 2005 , retention periods still vary significantly across States, ranging from two years to indefinitely. Such considerable differences between programs is reflective of the situation internationally where there is no agreement on the appropriate retention period of newborn blood samples. ${ }^{2,7}$ Closely associated with sample retention periods is the issue of what, if any, secondary uses are appropriate for the blood samples collected for the sole purpose of newborn screening.

While sample retention for forensic and quality assurance purposes, and for the development or modification of screening tests is justifiable, it is the potential for these samples to be used to conduct population based health screening studies and epidemiological research that prompts the need to articulate agreed standards in this area. It is very difficult to obtain population datasets of this kind that derive from the whole population with no selection. ${ }^{7}$ Consequently, the research opportunities are plentiful. In the United Kingdom, the value of conducting research based on blood samples gathered through newborn screening is acknowledged as having contributed towards answering important public health questions and leading to advancements in newborn and antenatal screening technologies. ${ }^{8}$

At the same time, significant ethical issues underlie the ability to access blood samples derived from newborn screening, including the appropriateness of using samples obtained through dissent rather than informed consent for secondary purposes, ${ }^{2}$ and the ability to garner public support for such research. Biological specimen databanks are often met with great reservation by the public because of their potential for misuse and a lack of visible bodies to provide regulation. ${ }^{7}$ Further, these ethical issues may vary according to the levels of access researchers would be afforded, in particular whether they would have access to identified or de-identified samples.

Ascertaining the views of the community, and parents in particular, about the retention and use of newborn blood samples forms a critical component of the development of any policy in this area. Such studies are a growing area of investigation within the psychology of newborn screening, ${ }^{9}$ although not yet explored extensively. To date these studies have targeted the parent's levels of knowledge about the programs and any psychosocial issues related to the provision of positive test results for particular disorders. ${ }^{10111213}$ It is reasonable to anticipate that the attitudes of parents whose 
child has been diagnosed through newborn screening may be different from those parents whose child was found not to have an inborn error of metabolism, or from the community in general.

Although several factors influence parental views about newborn screening, parents have been found to be generally supportive of newborn screening programs, even in the case where false-positive test results have been given. ${ }^{4}$ Positive attitudes are not necessarily contingent upon adequate knowledge of screening, as there is substantial evidence suggesting that parents often have limited knowledge of which disorders are tested for, the effects of the disorders and the treatments available. ${ }^{9}$ Many new mothers are not even aware of the test being performed on their child. ${ }^{14}$ Further, a recent study has found that parents in the United States are not well informed about the storage of cards, or their potential uses, and only five percent of educational materials aimed at informing parents about newborn screening actually address these issues. ${ }^{15}$ Although data regarding attitudes towards storage and use of newborn screening samples is limited, Gustafsson Stolt et al (2002) report their respondents expressing concern about the storage of material and the right to be informed of any screening or project results. ${ }^{16}$

In relation to using newborn blood samples for research purposes, data from Sweden suggests that mothers have generally positive attitudes to research. Those who choose to allow their child's blood sample to be used for research cite the potential to contribute to research as the primary motivation for doing so. ${ }^{16}$ Those who do not allow participation often cite concerns about making decisions on behalf of their child regarding genetic material as a primary consideration. ${ }^{16}$

The paucity of information about parental attitudes in relation to issues in newborn screening, ${ }^{13}$ has been identified as an issue requiring exploration. For policy development in particular, greater dialogue between government and community is necessary so that community concerns and any associated ethical issues may be adequately addressed. ${ }^{7,16}$ This study aims to explore new mothers' knowledge and attitudes towards newborn genetic blood screening. Specifically, it aims to ascertain new mothers' awareness of newborn screening, and their attitudes about issues relating to the retention and use of blood samples to include research purposes in Western Australia.

\section{Methods}

\section{Participants}

Women who gave birth during the month of January 2005 were invited to participate. Those women who gave birth to a stillborn child or those whose child died neonatally were excluded.

\section{Measures}

Information was collected via a self-administered mail survey, which women received four month's after the birth of their child. Items included in the survey were constructed following a review of the literature. Further, the investigators met with a group of new mothers to ask them about their experience with newborn blood screening to inform survey construction. A pilot study was also conducted to verify ease of understanding. 
The survey investigated four key areas, namely awareness of newborn screening, attitudes about appropriate retention periods for samples, attitudes towards the use of samples for secondary purposes specifically research, and demographic information. Participants were also given the opportunity to provide comments in order to enable aspects of these issues, which could not be explored through structured questions to be investigated.

\section{Procedure}

Women meeting the inclusion criteria were selected from the Midwives Notification Database located at the Department of Health (Western Australia). These women were sent a copy of the survey, along with an information sheet detailing the method of participant selection, the purpose of the study, background information on newborn screening, and a reply paid envelope. Women were informed that completion and return of the survey was deemed consent to participate.

The University of Western Australia Human Research Ethics Committee approved the study and the Confidentiality in Health Information Committee at the Department of Health, Western Australia granted access to the Midwives Notification Database.

\section{Data Analysis}

Descriptive statistics, Spearman's Rank Order Correlation multiple response analysis procedures were used to analyse the data.

\section{Results}

The survey was sent to 1846 new mothers, of which 600 returned a completed survey, equating to a response rate of $33 \%$. Although this response rate is typical for a selfcompleted mail survey, ${ }^{17}$ and for new mothers in particular, ${ }^{18}$ an independent group of new mothers was sampled through seven new mother's groups in the metropolitan area to check for bias in the study cohort. All new mothers present completed a short version of the mail survey $(\mathrm{N}=52)$. Demographic data were not collected on these new mothers. Responses from this sample were consistent with those obtained in the main survey.

Ages ranged from 18 to 47 years with the mean age of the sample being 32 years. The number of children the women had ranged from one child to seven, with the average being two children. First time mothers comprised $44 \%$ of the sample. The majority of women lived within the metropolitan area (78\%). Over a third of women had a university education (36\%), and $27 \%$ reported that they had post-secondary school qualifications.

To determine the representativeness of the sample, data from the Midwives Notification Database on all women who gave birth during January 2005 was obtained. Table 1 illustrates the differences between the population group and the sample collected. 
Table 1. Comparison of population and sample demographics

\begin{tabular}{lll}
\hline & Population $(\mathbf{N}=\mathbf{1 9 4 5})$ & Sample $(\mathbf{N}=\mathbf{6 0 0})$ \\
\hline Mean age & 29.4 years & 31.9 years \\
$\begin{array}{l}\text { Mean number of children } \\
\text { Geographical area }\end{array}$ & 2 & \\
Metropolitan & $74 \%$ & $78 \%$ \\
Rural & $26 \%$ & $22 \%$ \\
& & \\
\hline
\end{tabular}

\section{Awareness of Newborn Genetic Blood Screening}

The majority of women (93\%) stated that they had heard of newborn genetic blood screening prior to receiving the survey. They reported receiving this information from a variety of sources (Table 2 ).

Table 2. Respondent's reported sources of information about newborn screening

\begin{tabular}{ll}
\hline Sources of information & Most popular sources reported (\%) \\
\hline Midwife & $34 \%$ \\
Previous pregnancy & $27 \%$ \\
Newborn screening pamphlet & $14 \%$ \\
General practitioner or & $12 \%$ \\
$\quad$ obstetrician & $12 \%$ \\
Family or friends & $1 \%$ \\
Internet & \\
\hline
\end{tabular}

Over half of all respondents were satisfied with the information provided (51\%), with the remainder reporting higher (19\%) or lower levels of satisfaction (18\%). When provided with the opportunity to comment further, the women stated that the time at which the information was provided was a significant factor in determining their ability to adequately consider the information:

I feel the test isn't discussed enough at the time it is done. A midwife takes your baby, does the test, brings baby back and leaves a pamphlet that on most occasions gets put aside with all that's going on.

(The information) could have been lost in avalanche of other information and emotion.

Women also reported that they would have liked to receive more comprehensive information:

Only very general information was given to me when the tests were done. I would have liked more detailed information (from midwife or information booklets).

I don't believe the information I received from my GP, Obstetrician, or hospital was adequate. 
In order to gauge the extent to which women valued newborn genetic blood screening, they were asked to rate their agreement with the following statement: 'I believe newborn blood screening is valuable for enabling the early detection of genetic disorders in children'. The majority either strongly agreed (61\%) or agreed (34\%). Only 5\% stated that they either disagreed or held no opinion. Women who had received information about newborn screening are more likely to value the screening test $(.099, \mathrm{p}=.017)$ and women's belief in the value of newborn screening positively correlated with the degree to which they are satisfied with the information they were provided $(.370, \mathrm{p}=.000)$.

\section{Newborn Blood Sample Retention Periods}

Women were asked to nominate an appropriate time-period for retaining newborn blood samples before they are destroyed (Table 3).

Table 3. Support for proposed newborn screening blood sample retention periods

\begin{tabular}{lc}
\hline Period of Retention & Percentage of Respondents \\
\hline 2 years or less & $29.3 \%$ \\
$3-10$ years & $29.5 \%$ \\
$11-20$ years & $8.6 \%$ \\
21 years or more & $16.8 \%$ \\
Indefinitely & $0.6 \%$ \\
Unsure & $6.3 \%$ \\
As long as they are required & $8.9 \%$ \\
\hline
\end{tabular}

When asked to nominate reasons for their choice, women who believed in maintaining the retention period of two years spoke of not possessing adequate knowledge of the issues involved to justify deviating from current practice:

I do not know a lot about the screening test nor have heard of reasons why they may be kept longer. Perhaps if I had more information my answer may be different.

I don't know enough about the information gathered from the screening to believe the timeframe should be different from the present period of two years.

Other reasons for maintaining the current practice two-year retention period included statements that the time was adequate for the primary purpose of the test to be achieved and that this short period of time is a safeguard against any unnecessary research:

My understanding is the disorders checked for occur in infancy... and two years should be adequate to gather data for any research needs.

(Two years is appropriate) so information is gathered promptly and cards don't just sit there waiting for someone to come up with something new to research. 
Some women also distinguished between the results of the tests, believing that a longer period of retention could be justifiable for those samples, which test positive to enable research.

Women who nominated between 3-10 years as being an appropriate retention period were keen to promote research on the samples, believing that this time enabled more research to take place. They also reported the belief that this period allowed for advancements in technology to enable the cards to be re-checked if necessary:

It could provide a reference point if the child develops any problems beyond two years, plus it may be useful for future research which should be automatic unless parent specifies otherwise.

The longer retention periods of cards was also nominated because it is in keeping with standard record retention periods of between 5 to 10 years for important documentation, such as those required for taxation purposes.

There was also support for a retention period of 21 years or longer (17\%), the advantage of which was the facilitation of research opportunities.

\section{Attitudes towards the Use of Newborn Blood Samples for Research Purposes}

Most of the cohort (85\%) believed that de-identified newborn screening samples should be made available for research, the remainder either disagreed $(4 \%)$ or were unsure $(11 \%)$.

In order to gain an appreciation for the strength of women's belief in the use of cards for research, they were asked the extent to which they agreed with the statement " $I$ would agree to my baby's card being used for research". It was found that $85 \%$ of respondents either strongly agreed or agreed with the statement. A further $9 \%$ held no opinion, and 5\% disagreed. In addition, 79\% either strongly agreed or agreed with the statement "I would like to have the opportunity to contribute positively to research through newborn screening cards". This sense of support for research was also reflected in the comments:

Whatever information can be collected and research done on cards can only be of a benefit to medical community to better understand genetic diseases or other diseases that are influenced by lifestyle / environment.

The women viewed de-identification of blood samples as a priority. It was found that when questioned about the importance of de-identifying blood samples for any purpose other than newborn screening, $90 \%$ of the sample agreed that this should occur. The importance of de-identification was also demonstrated through the comments provided:

I am happy for my child's blood screening test to be used for research... as long as my child's identity and personal information is removed. 
I feel strongly about ensuring that genetic details and information is kept private. Guaranteed privacy of genetic information would be essential to my support of any research.

Closely associated with this is their belief that parental consent should be sought prior to the samples being made available for research purposes:

I feel strongly that consent should have to be given before samples are used for any purpose other than the specified genetic tests.

Additionally, women were keen to receive information about the types of research that may be conducted so that they could make an informed decision as to whether the research is compatible with their ethical values:

If this information was used for research I would like to know what type of research to decide if I agreed with it ethically and morally.

I would be fine with use of sample for research if it was specifically outlined what the use would be and how the sample would be stored / destroyed / managed.

In order to ascertain if women's attitudes would be dependent upon the type of research that the samples could be used for, four research areas were listed and women were asked to nominate the extent to which they felt using the samples would be appropriate. These areas were (i) to understand the prevalence of disease in the community, (ii) to improve diagnostic tests for childhood diseases, (iii) to understand how lifestyle factors influence genetic diseases, and (iv) to improve diagnostic testing of diseases. All four areas were overwhelmingly supported. The most support was given to improving diagnostic tests for childhood diseases (97\%), followed by improving diagnostic testing (96\%), understanding prevalence of disease $(93 \%)$, and lifestyle and genetic interactions (92\%).

When asked to nominate any types of research that would be unacceptable uses of newborn screening cards, the fields of uses most commonly cited were cloning, research that leads to abortion, 'designer babies', and the use of the information for paternity issues or criminal investigations.

Further, women noted the need for necessary safeguards against inappropriate use to be in place prior any changes to current practice:

I would be happy for my child's card to be used for research but have concerns regarding the safety of personal and genetic information, and the relevance of the research. I wouldn't want the cards kept for the purposes of doing research that does not directly benefit the community.

Ethics is an important issue here and the availability of data to insurance companies, other family members, police etc without consent or by law is a problem not yet dealt with.

\section{Other Relationships}

No relationships between demographic factors and outcomes on the key variables were found. 


\section{Discussion}

This study investigated knowledge and attitudes of new mothers who had received no further information about newborn screening from the researchers, other than that presented in the newborn screening pamphlet, prior to completing the survey. In this way, the results generated provide a sound indication of the views of new mothers in Western Australia about issues relating to newborn screening and the potential use of screening samples for secondary purposes.

The results suggest that women are aware of newborn screening, but do not feel that they are well informed. This echoes previous research indicating that new mothers possess limited awareness of the newborn screening test being performed, ${ }^{14}$ and its purpose, including the diseases being tested. ${ }^{9,19}$ Most women received screening information within the 72-hour period after the birth of their child. During this time, they receive a plethora of information relating to other aspects of neonatal care. It is also common for midwives to perform the blood test away from the mother to circumvent any unnecessary anxiety, ${ }^{14}$ so it is not surprising that women may have difficulty assimilating screening information for a test for which they must 'opt-out'.

Previous studies have shown that the time at which screening information is provided was a critical determinant of women's ability to adequately absorb the information. Therefore it may be more beneficial for health professionals to endeavour to provide this information during the prenatal period. ${ }^{2,15}$ In addition to facilitating the comprehension of the material, presenting information at this time would also enable more detail to be passed onto parents, and provide an opportunity for questions to be asked. Women were keen to receive additional newborn screening information through government pamphlets. Only a small proportion of women reported reading this pamphlet, and therefore it may be useful to ascertain if other forms of communicating this information may be more relevant for this group.

Ensuring that women have adequate time to consider screening information becomes even more salient when considering the possibility of research on newborn blood samples. There was considerable support for the use of blood samples for research, and this support was motivated by the desire to make a positive contribution to research, mirroring results found for a similar cohort overseas. ${ }^{16}$ The women were also particularly supportive of research targeted at child health issues, indicating a belief that research on stored samples should be closely aligned with the primary purpose of the sample collection. However, support for research was contingent upon safeguards against breaches to privacy and policy makers need to be mindful of the public's wariness of biobanks and ensure that the appropriate safeguards are in place prior to the systematic storage of newborn blood samples for extended periods.

In relation to retention periods, the strong support of maintaining the current WA storage period of two years is to be expected. Without a thorough knowledge of the issues, determining a justification for nominating a longer time-period is difficult. Nevertheless, the women equally supported storage of between three to ten years to allow adequate time for research. The qualitative data also suggests that women are generally uncomfortable with the long-term retention of cards for research purposes. These results suggest that given appropriate justification, samples may be stored for 
longer than the current two-year period, although storage beyond a decade is unsupported.

There were specific aspects arising from this research which merit further investigation. In particular, this study did not collect information on whether any of the women participating had received either a positive or a false positive test result. Although the expected numbers for such results would be low, it would nevertheless be valuable to explore any differentiation between these groups from the core group across all variables. Secondly, the investigation of women's awareness of screening could be explored in greater detail to gain an indication of women's actual knowledge levels, for example, their knowledge of what conditions are screened for in testing.

\section{Acknowledgements}

The authors would like to thank all new mothers who participated in this study, Ms Vivien Gee, Manager of the Maternal and Child Health Unit, Department of Health Western Australia and also Ms Nikki Breheny and Ms Taryn Charles who assisted with the data collection and analysis.

\footnotetext{
${ }^{1}$ M. Metz et al. Newborn screening in South Australia: is it universal? MJA 2003; 179: 412-415.

2 L. Kharaboyan et al. Storing newborn blood spots: Modern Controversies. Intl Comp Health Law Ethics 2004; 32, 4: 741-748.

3 D. Ciske et al. Genetic counseling and neonatal screening for cystic fibrosis: an assessment of the communication process. Pediatrics 2001; 107, 4: 699-705.

4 E. Parsons et al. Psychosocial issues in newborn screening for cystic fibrosis. Paediatr Respir Rev 2003; 4: 285-292.

5 P. O'Leary et al. Storage of Newborn Screening Cards: Legal and Ethical Issues: Genomics Directorate, Department of Health (WA). 2004.

${ }^{6}$ E. Geelhoed et al. An economic evaluation of neonatal screening for phenylketonuria and congenital hypothyroidism. J Paediatr Child Health in press.

7 B. Norgaard-Pedersen et al. Biological specimen banks in neonatal screening. Acta Paediatr Suppl 1999; 432:106-109.

${ }^{8}$ Policies and standards for newborn blood spot screening: UK Newborn Screening Program Centre. 2005.

${ }^{9}$ R. Pollitt et al. Neonatal screening for inborn errors of metabolism: cost, yield and outcome. Health Technol Assess 1997; 1, 7: 1-202.

${ }^{10}$ D. Axworthy et al. Psychological impact of population-based carrier testing for cystic fibrosis: 3year follow-up. Lancet 1996; 347: 1443-1446.

${ }^{11} \mathrm{C}$. Kwon et al. The magnitude and challenge of false-positive newborn screening test results. Arch Pediatr Adolesc Med 2000; 154, 7: 714-718.

12 E. Parsons et al. Newborn screening for Duchenne muscular dystrophy: A psychosocial study. Arch Dis Child Fetal Neonatal Ed 2002; 86: 91-95.

13 J. Green et al. Psychosocial aspects of genetic screening of pregnant women and newborns: a systematic review. Health Technol Assess 2004; 8, 33:1-109.

${ }^{14}$ C. Suriadi et al. Factors affecting mothers' knowledge of genetic screening. Aust N Z J Obstet Gynaecol. 2004; 44:30-34.

${ }^{15} \mathrm{~K}$. Fant et al. Completeness and complexity of information available to parents from newborn screening programs. Pediatrics 2005; 115, 5: 1268-1272.

${ }^{16}$ U. Gustafsson Stolt et al. Attitudes to bioethical issues: A case study of a screening project. Soc Sci Med 2002; 54: 1333-1344.
} 
17 D. Martin. Doing Psychology Experiments. Brooks/Cole Publishing Company, CA, United States, 1996.

${ }^{18}$ K. Rostant et al. Survey of the knowledge, attitudes and experiences of Western Australian women in relation to prenatal screening and diagnostic procedures. Aust N Z J Obstet Gynaecol 2003; 43, 2: 134138.

${ }^{19}$ K. Hargreaves et al. Informed choice and public health screening for children: the case of blood spot screening. Health Expectations 2005; 8:161-171 